\title{
HYPERSURFACES OF ODD-DIMENSIONAL SPHERES
}

\author{
DAVID E. BLAIR, GERALD D. LUDDEN \& KENTARO YANO
}

A structure similar to an almost complex structure was shown in [2] to exist on a hypersurface of an almost contact manifold or a submanifold of codimension 2 of an almost complex space. This structure on a manifold $M$ has been studied in [1], [5], [6] from two points of view, namely, that the structure exists on $M$ because $M$ is a submanifold of some ambient space, and also that the structure exists intrinsically on $M$.

The odd-dimensional sphere $S^{2 n+1}$ has an almost contact structure which is naturally induced from the Kaehler structure of Euclidean space $E^{2 n+2}$. The purpose of this paper is to study complete hypersurfaces immersed in $S^{2 n+1}$. In $\S 3$ it is shown that if the Weingarten map of the immersion and $f$ commute then the hypersurface is a sphere whose radius is determined. Here, $f$ is a tensor field of type $(1,1)$ on the hypersurface, which is part of the induced structure. That the hypersurface satisfying this condition is a sphere follows from the results in [6], however a new proof is given here for completeness. In $\S 4$ it is shown that if the Weingarten map $K$ of the immersion and $f$ satisfy $f K+K f=0$, and the hypersurface is of constant scalar curvature, then it is a great sphere or $\mathrm{S}^{n} \times S^{n}$.

\section{Hypersurfaces of a sphere}

Let $S^{2 n+1}$ be the natural sphere of dimension $2 n+1$ in Euclidean $(2 n+2)$ space $E^{2 n+2}$. Let $(\phi, \xi, \eta, g)$ be the normal, almost contact metric structure (see [4]) induced on $S^{2 n+1}$ by the Kaehler structure on $E^{2 n+2}$. That is to say, $\phi$ is a tensor field of type $(1,1), \xi$ is a vector field, $\eta$ is a 1 -form and $g$ is a Riemannian metric on $S^{2 n+1}$ satisfying

$$
\begin{gathered}
\phi^{2}=-I+\eta \otimes \xi, \\
\phi \xi=0, \quad \eta \circ \phi=0, \\
\eta(\xi)=1, \\
g(\phi \bar{X}, \phi \bar{Y})+\eta(\bar{X}) \eta(\bar{Y})=g(\bar{X}, \bar{Y}), \\
{[\phi, \phi]+d \eta \otimes \xi=0,}
\end{gathered}
$$

where $[\phi, \phi]$ is the Nijenhuis torsion tensor of $\phi$, and $\bar{X}$ and $\hat{Y}$ are arbitrary vector fields on $S^{2 n+1}$.

Communicated December 17, 1970. 
Suppose $\pi: M^{2 n} \rightarrow S^{2 n+1}$ is an embedding of the orientable manifold $M^{2 n}$ in $S^{2 n+1}$. The tensor $G$ defined on $M^{2 n}$ by

$$
G(X, Y)=g\left(\pi_{*} X, \pi_{*} Y\right)
$$

is a Riemannian metric on $M^{2 n}$, where $\pi_{*}$ denotes the differential of the embedding $\pi$. If $C$ is a field of unit normals defined on $M^{2 n}$, and $\tilde{\nabla}$ is the Riemannian connection of $g$, then the Gauss and Weingarten equations can be written as

$$
\begin{aligned}
\tilde{\nabla}_{\pi_{*} X} \pi_{*} Y & =\pi_{*}\left(\nabla_{X} Y\right)+k(X, Y) C \\
\tilde{\nabla}_{\pi_{*} X} C & =\pi_{*}(K X) .
\end{aligned}
$$

Then $\nabla$ is the Riemannian connection of $G, k$ is a symmetric tensor of type $(0,2)$ on $M^{2 n}$, and $G(K X, Y)=k(X, Y)$. Furthermore, if we set

$$
\begin{aligned}
\phi \pi_{*} X & =\pi_{*} f X+v(X) C, \quad \xi=\pi_{*} U+\lambda C, \\
\phi C & =-B V, \quad u(X)=\eta\left(\pi_{*} X\right),
\end{aligned}
$$

then $f$ is a tensor field of type $(1,1), U$ and $V$ are vector fields, $u$ and $v$ are 1 -forms, and $\lambda$ is a function satisfying

$$
\begin{aligned}
f^{2} & =-I+u \otimes U+v \otimes V, \\
u \circ f & =\lambda v, \quad v \circ f=-\lambda u, \\
f U & =-\lambda V, \quad f V=\lambda U, \\
u(U) & =v(V)=1-\lambda^{2}, \quad u(V)=v(U)=0, \\
G(f X, f Y) & =G(X, Y)-u(X) u(Y)-v(X) v(Y) .
\end{aligned}
$$

It was shown in [2] that the following relations hold

$$
\begin{aligned}
\left(\nabla_{X} f\right) Y & =G(X, Y) U-u(Y) X-k(X, Y) V+v(Y) K X \\
\nabla_{X} U & =-f X-\lambda K X \\
\nabla_{X} V & =-\lambda X+f K X \\
\nabla_{X} \lambda & =v(X)+k(U, X) .
\end{aligned}
$$

\section{Case I: $K f-f K=0$}

We will prove the following theorem.

Theorem 1. If $M^{2 n}$ is an orientable submanifold of $S^{2 n+1}$ satisfying $K f=f K$, and $\lambda \neq$ constant, $K$ being the Weingarten map of the embedding, and $f$ and $\lambda$ being defined in (4), then $M^{2 n}$ is a sphere of radius $1 / \sqrt{1+\alpha^{2}}$, where $\alpha$ is some constant determined by the embedding. 
Proof. We have that $0=G((K f-f K) U, U)$, so that

$$
\begin{aligned}
0 & =G(K f U, U)-G(f K U, U) \\
& =-\lambda G(V, K U)+G(K U, f U) \\
& =-\lambda G(V, K U)-\lambda G(K U, V) .
\end{aligned}
$$

Therefore we see $\lambda=0$ or $k(U, V)=0$. By continuity, since $\lambda$ is non-constant,

$$
k(U, V)=0 .
$$

In a similar fashion we obtain

$$
k(U, U)=k(V, V) .
$$

Now $f K U+\lambda K V=0$, so that

$$
\begin{aligned}
0 & =-K U+u(K U) U+v(K U) V+\lambda K f V \\
& =-K U+u(K U) U+\lambda^{2} K U,
\end{aligned}
$$

and hence

$$
\left(1-\lambda^{2}\right) K U=k(U, U) U
$$

Similarly, we obtain

$$
\left(1-\lambda^{2}\right) K V=k(U, U) V .
$$

At points where $\lambda \neq \pm 1$, we have $K U=\alpha U$ for $\alpha=k(U, U) /\left(1-\lambda^{2}\right)$, which implies

$$
\left(\nabla_{X} K\right) U+K(-f X-\lambda K X)=\nabla_{X} \alpha \cdot U+\alpha(-f X-\lambda K X) .
$$

The Codazzi equation for an embedding gives that $\left(\nabla_{X} K\right)(Y)=\left(\nabla_{Y} K\right)(X)$ so that we have

$$
2 G(K f Z, X)=\left(\nabla_{X} \alpha\right) u(Z)-\left(\nabla_{Z} \alpha\right) u(X)+2 \alpha G(f Z, X) .
$$

If we set $Z$ equal to $U$, then

$$
-2 \alpha \lambda u(X)=\left(\nabla_{X} \alpha\right)\left(1-\lambda^{2}\right)-\left(\nabla_{U} \alpha\right) u(X)-2 \lambda u(X),
$$

so that $\nabla_{X} \alpha$ and $u(X)$ are proportional. Therefore $K f=\alpha f$, and hence

$$
-K X+u(X) K U+v(X) K V=\alpha(-X+u(X) U+v(X) V) .
$$

Thus $K X=\alpha X$ for all $X$, and by the Codazzi equation $\alpha$ is constant. From (6) we have that $V_{X} \lambda=v(X)+\alpha u(X)$, and therefore that 


$$
\begin{aligned}
\nabla_{Y} \nabla_{X} \lambda-d \lambda\left(\nabla_{Y} X\right) & =\nabla_{Y}(v(X)+\alpha u(X))-\left(v\left(\nabla_{Y} X\right)+\alpha u\left(\nabla_{Y} X\right)\right) \\
& =-\lambda G(X, Y)+\alpha G(X, f Y)-\alpha G(X, f Y)-\alpha \lambda G(K Y, X) \\
& =-\lambda\left(1+\alpha^{2}\right) G(X, Y) .
\end{aligned}
$$

By the following lemma of Obata [3], $M^{2 n}$ is sphere of radius $\left(1+\alpha^{2}\right)^{-1 / 2}$.

Lemma. A complete connected Riemannian manifold $M$ admits a nontrivial solution $\lambda$ of $\nabla_{Y} \nabla_{X} \lambda-d \lambda\left(\nabla_{Y} X\right)=-k \lambda G(X, Y)$ for some real number $k>0$ if and only if $M$ is globally isometric to a Euclidean sphere of radius $k^{-1 / 2}$.

Corollary. Let $M^{2 n}$ be an orientable submanifold of $S^{2 n+1}$ with $\lambda \neq$ constant. Then $K f-f K=0$ if and only if $M^{2 n}$ is a totally umbillical submanifold of $S^{2 n+1}$.

Remark. In [5], there was introduced the idea of normality of an $(f, G, u$, $v, \lambda$ )-structure, which is of a manifold $M^{2 n}$ with tensors satisfying (5). This condition is

$$
[f, f]+d u \otimes U+d v \otimes V=0 .
$$

We have the following proposition.

Proposition. Let $M^{2 n}$ be a hypersurface of $S^{2 n+1}$ with $\lambda \neq$ constant. The $(f, G, u, v, \lambda)$-structure on $M^{2 n}$ is normal if and only if $f K-K f=0$.

Proof. Let

$$
S(X, Y)=[f, f](X, Y)+d u(X, Y) U+d v(X, Y) V .
$$

Using (5) it can be shown that

$$
S(X, Y)=v(Y)(K f-f K) X-v(X)(K f-f K) Y,
$$

and hence the "if" part of the proposition is proved. On the other hand, assume $S(X, Y)=0$ for all $X$ and $Y$ and let $P X=(K f-f K) X$. Then

$$
v(V) P X=v(X) P V .
$$

Also, it can be shown that

$$
G(P X, Y)=G(X, P Y)
$$

so that

$$
v(X) G(P V, Y)=v(Y) G(P V, X),
$$

that is to say,

$$
G(P V, Y)=\alpha v(Y)
$$

for some $\alpha$. Thus we have that 


$$
v(V) G(P X, Y)=v(X) G(P V, Y)=\alpha v(X) v(Y),
$$

but since the trace of $P$ is 0 , we have $\alpha=0$ and thus $P=0$.

\section{Case II: $K f+f K=0$}

In this section we prove the following theorem.

Theorem 2. If $M^{2 n}$ is a complete orientable submanifold of $S^{2 n+1}$ with constant scalar curvature satisfying $K f+f K=0$ and $\lambda \neq$ constant, where $K$ is the Weingarten map of the embedding, and $f$ and $\lambda$ are defined in (4), then $M^{2 n}$ is a natural sphere $S^{2 n}$ or $M^{2 n}=S^{n} \times S^{n}$.

Proof. We have that

$$
\begin{aligned}
& 0=(K f+f K) U=-\lambda K V+f K U, \\
& 0=(K f+f K) V=\lambda K U+f K V,
\end{aligned}
$$

so that

$$
\begin{aligned}
0 & =-\lambda k(V, V)+G(f K U, V) \\
& =-\lambda k(V, V)-G(K U, f V) \\
& =-\lambda k(V, V)-\lambda k(U, U)
\end{aligned}
$$

and hence

$$
k(U, U)+k(V, V)=0
$$

by continuity. Also

$$
\begin{aligned}
0 & =-\lambda f K V+f^{2} K U \\
& =\lambda^{2} K U+(-K U+u(K U) U+v(K U) V),
\end{aligned}
$$

that is,

$$
\left(1-\lambda^{2}\right) K U=k(U, U) U+k(U, V) V
$$

and similarly

$$
\left(1-\lambda^{2}\right) K V=k(U, V) U+k(V, V) V .
$$

At points where $\lambda \neq \pm 1$, write equations (10) and (11) as

$$
\begin{aligned}
& K U=\alpha U+\beta V, \\
& K V=\beta U-\alpha V .
\end{aligned}
$$

If we apply $\nabla_{X}$ to equation (10'), use equation (6) for $\nabla_{X} U$ and $\nabla_{X} V$, and use 
the fact that $\left(\nabla_{X} K\right) Y=\left(\nabla_{Y} K\right) X$ because of the Codazzi equation, then we find that

$$
\begin{aligned}
\left(\nabla_{X} \alpha\right) u(Y) & -\left(\nabla_{Y} \alpha\right) u(X)+\left(\nabla_{X} \beta\right) v(Y) \\
& -\left(\nabla_{Y} \beta\right) v(X)-2 \alpha F(X, Y)=0,
\end{aligned}
$$

where $F(X, Y)=G(f X, Y)$. Setting $X=U$ and $Y=V$ and using the fact that $\lambda \neq$ constant we see that

$$
-\nabla_{V} \alpha+\nabla_{U} \beta+2 \alpha \lambda=0 .
$$

From equations (12) and (13) we obtain

$$
\begin{gathered}
\left(1-\lambda^{2}\right) \nabla_{Y} \alpha=\left(\nabla_{U} \alpha\right) u(Y)+\left(\nabla_{V} \alpha\right) v(Y), \\
\left(1-\lambda^{2}\right) \nabla_{Y} \beta=\left(\nabla_{U} \beta\right) u(Y)+\left(\nabla_{V} \beta\right) v(Y), \\
2 \alpha\left(1-\lambda^{2}\right) F(X, Y)=(u(Y) v(X)-v(Y) u(X))\left(\nabla_{V} \alpha-\nabla_{U} \beta\right) .
\end{gathered}
$$

However, since the rank of $f$ is $\geq 2 n-2$, equation (16) implies that $\alpha=0$ and $\nabla_{U} \beta=0$ if $n \neq 1$. Thus equation (12) becomes

$$
\left(\nabla_{X} \beta\right) v(Y)=\left(\nabla_{Y} \beta\right) v(X)
$$

or

$$
\left(1-\lambda^{2}\right) \nabla_{X} \beta=\left(\nabla_{V} \beta\right) v(X) .
$$

Applying $\nabla_{X}$ to equation $\left(11^{\prime}\right)$, and using the fact that $\alpha=0$ and the Codazzi equation, we find that

$$
\left(\nabla_{X} \beta\right) u(X)-\left(\nabla_{Y} \beta\right) u(X)-2 \beta F(X, Y)-2 F(K X, K Y)=0 .
$$

Setting $Y=U$ and using $\left(12^{\prime \prime}\right)$ we have that $2 \beta^{2} \lambda=2 \beta \lambda-\nabla_{V} \beta$ so that $\beta=$ constant implies that $\beta=0$ or $\beta=1$.

Replace $Y$ by $f Y$ in equation (17) and use equation (12') to obtain

$$
\begin{aligned}
& 2\left(1-\lambda^{2}\right) F(K X, K f Y) \\
& =\left(\nabla_{V} \beta\right)(v(X) u(f Y)-v(f Y) u(X))-2 \beta\left(1-\lambda^{2}\right) F(X, f Y),
\end{aligned}
$$

that is,

$$
\begin{aligned}
-2\left(1-\lambda^{2}\right)[G(K X, K Y)-u(K X) u(K Y)-v(K X) v(K Y)] \\
=\nabla_{V} \beta[\lambda v(X) v(Y)+\lambda u(X) u(Y)] \\
-2 \beta\left(1-\lambda^{2}\right)[G(X, Y)-u(X) u(Y)-v(X) v(Y)],
\end{aligned}
$$

from which follows 


$$
\left(1-\lambda^{2}\right) K^{2}=\left(\beta^{2}-\beta\right)(u \otimes U+v \otimes V)+\beta\left(1-\lambda^{2}\right) I .
$$

From (18) and a previous remark we see that if $\beta=$ constant then $K^{2}=0$ or $K^{2}=I$. If $K^{2}=0$, then $K=0$ since $K$ is symmetric. In this case, $M^{2 n}$ is a totally geodisic submanifold of $S^{2 n+1}$ and hence $M^{2 n}=S^{2 n}$. In the case where $K^{2}=I, K$ gives an almost product structure on $M^{2 n}$.

We have

$$
\begin{aligned}
k(f X, f Y) & =G(K f X, f Y)=G\left(K f^{2} X, Y\right) \\
& =-G(K X, Y)+u(X) G(K U, Y)+v(X) G(K V, Y) \\
& =-k(X, Y)+\beta(u(X) v(Y)+v(X) u(Y))
\end{aligned}
$$

Now since $k(U, U)+k(V, V)=0$ and $G(U, V)=0$, the last equation can be used to show that the trace of $K$ is 0 , that is, $M^{2 n}$ is a minimal hypersurface (note that this last conclusion holds whether or not $\beta=$ constant). In the case where $K^{2}=I, \operatorname{tr} K=0$ implies that the global distributions on $M^{2 n}$ given by $\frac{1}{2}(K+I)$ and $\frac{1}{2}(I-K)$ are both of dimension $n$.

Now to find the scalar curvature of $M^{2 n}$ by the Gauss equation, let $\tilde{R}$ and $R$ denote the curvature tensors of $g$ and $G$ respectively. Then the Gauss equation is

$$
\begin{aligned}
& \tilde{R}\left(\pi_{*} X, \pi_{*} Y, \pi_{*} Z, \pi_{*} W\right) \\
& \quad=R(X, Y, Z, W)-(k(Y, Z) k(X, W)-k(Y, W) k(X, Z)) .
\end{aligned}
$$

Using (18) and the fact that $S^{2 n+1}$ is of constant curvature equal to 1 , for $1-\lambda^{2} \neq 0$ we have

$$
\begin{aligned}
\bar{R}(X, Y)= & (2 n-1) g(X, Y) \\
& -\left[\beta g(X, Y)+\frac{\beta^{2}-\beta}{1-\lambda^{2}}(u(X) u(Y)+v(X) v(Y))\right],
\end{aligned}
$$

where $\bar{R}$ is the Ricci tensor of $G$. From this it follows that the scalar curvature of $M^{2 n}$ is equal to $2 n(2 n-1)-\beta(2 n-2)-2 \beta^{2}$, and therefore that $\beta=$ constant.

If we apply $\nabla_{X}$ to equation (19) and use the second Bianchi identity and tr $K=0$, then we obtain that

$$
\left(\nabla_{X} K\right) Y+\left(\nabla_{Y} K\right) X=0
$$

and thus $\nabla_{X} K=0$ by the Codazzi equation.

Therefore, if $\beta=1$, the almost product structure $K$ is decomposable. Hence by completeness, $M^{2 n}$ is a product $M^{n} \times \bar{M}^{n}$. Now we have, by equation (17), 


$$
\begin{aligned}
& G(f(K \pm I) X,(K \pm I) Y) \\
& \quad=F(K X, K Y)+F(X, Y) \pm(F(K X, Y)+F(X, K Y))=0
\end{aligned}
$$

and, by equation (6),

$$
\begin{aligned}
\nabla_{Y} \nabla_{X} \lambda-d \lambda\left(\nabla_{Y}(X)\right) & =\nabla_{Y}(v(X)+k(U, X))-\left(v\left(\nabla_{Y} X\right)+k\left(U, \nabla_{Y} X\right)\right) \\
& =\left(\nabla_{Y} v\right) X+k\left(\nabla_{Y} U, X\right)=-2 \lambda G(X, Y)-2 G(f Y, K X) .
\end{aligned}
$$

From equation (20) we see that if $X$ and $Y$ are both in the distribution $I+K$ or $I-K$, then $g(f Y, K X)=0$ so that

$$
\nabla_{Y} \nabla_{X} \lambda-d \lambda\left(\nabla_{Y} X\right)=-2 \lambda G(X, Y) .
$$

Thus, $M^{n}$ and $\bar{M}^{n}$ are both spheres of radius $1 / \sqrt{2}$ by the lemma of Obata.

\section{References}

[ 1] D. E. Blair \& G. D. Ludden, On Intrinsic structures similar to those on $S^{2 n}$, Kōdai Math. Sem. Rep.

[ 2 ] D. E. Blair, G. D. Ludden \& K. Yano, Induced structures on submanifolds, Kōdai Math. Sem. Rep. 22 (1970) 188-198.

[3] M. Obata, Certain conditions for a Riemannian manifold to be isometric with a sphere, J. Math. Soc. Japan 14 (1962) 333-340.

[ 4] Y. Tashiro, On contact structure of hypersurfaces in complex manifolds. I, Tôhoku Math. J. 2 (1963) 62-78.

[ 5 ] K. Yano \& M. Okumura, On ( $f, g, u, v, \lambda)$-structures, Kōdai Math. Sem. Rep. 22 (1970) 401-423.

[6] _- On normal ( $f, g, u, v, \lambda)$-structures on submanifolds of codimension 2 in a Euclidean space, to appear.

Michigan State University 